\title{
INTRODUCTION
}

\section{A Stem Cell Primer}

\author{
JAMES F. BATTEY, JR. AND LAURA K. COLE
}

National Institute on Deafness and Other Communication Disorders [J.F.B., L.K.C.], National Institutes of Health, Bethesda, MD 20892

Why are scientists so interested in stem cells? Stem cells offer the hope of helping to advance science in many areas, including understanding more about the process of human development, creating new ways to treat disease and repair damaged tissues, and enabling us to test new drugs on cells of human origin without testing them on humans or animals.

Studying stem cells will help us to understand how a fertilized egg divides to produce the wide variety of specialized cells that make up the human body. Some of our most devastating medical conditions, such as cancer and birth defects, are due to problems with control of cell division. If scientists can better understand normal cell development, we may one day be able to correct the errors that cause these medical conditions.

Stem cells may also help scientists develop new ways to treat devastating illnesses and injuries. Although doctors today can use donated organs and tissues to replace those that are diseased or damaged, the demand from people needing a transplant far exceeds the supply of organs available for transplantation. If scientists can direct stem cells to become specialized tissues, they will have access to a renewable source of replacement cells and tissues to treat diseases and disabilities. Laboratories around the world are currently attempting to develop ways to use stem cells to treat Parkinson's disease, spinal cord injury, burns, heart disease, diabetes, and arthritis. Although most of these therapies are currently being studied in animal models, they offer hope for the future.

Scientists can use stem cells to test new drugs. They might, for example, test the safety of a new kidney drug on kidney tissue developed from human stem cell lines. Scientists are already using other kinds of cell lines for this purpose. Cancer cell lines, for example, are commonly used to screen potential anti-tumor drugs. Stem cells would increase scientists' abilities by allowing them to test drugs on a wider range of human cell types. Using human stem cells may also reduce the number of drugs that are tested on animals.

Before stem cells can be used to treat human disease or to test new drugs, scientists must learn how to reliably coax them into becoming the needed cell type-a process called differentiation. Once scientists have mastered the process of stem cell

Received November 16, 2005; accepted January 24, 2006.

Correspondence: James F. Battey, Jr., M.D., Ph.D., NIDCD/NIH, Building 31, Room 3C02, Bethesda, MD 20892; E-mail: batteyj@nidcd.nih.gov

DOI: 10.1203/01.pdr.0000208976.01669.c8 differentiation, many doors will open for medical research. As with any area of scientific investigation, the success of medical therapies developed from stem cells must be built upon a foundation of basic research discoveries. In this case, scientists must first understand the basic biology of stem cells and what gives them their special abilities.

\section{WHAT MAKES THESE CELLS SPECIAL?}

Stem cells have three very important properties that make them unique and useful: they can self-renew, they are pluripotent, and they can remain undifferentiated. Stem cells selfrenew when they divide to make more stem cells. Stem cells are also undifferentiated. That is, they do not have any characteristics of a specific cell type, such as the ability to "beat", as heart muscle cells do, or to transmit a nerve impulse, as nerve cells do. However, when the body is damaged by disease or injury, stem cells serve as a sort of reserve "pool" of repair cells. They can be called into service by distress signals transmitted by damaged tissues. The signals cause the unspecialized stem cells to begin to differentiate-that is, they assume characteristics of a specific tissue.

Based on their origin, stem cells may be divided into two very broad categories-embryonic stem cells and nonembryonic stem cells. The two categories differ in the degree of their unique stem-cell like abilities. In general, embryonic stem cells, derived from a very early stage in development, have greater abilities to self-renew, remain pluripotent, and remain undifferentiated. Nonembryonic stem cells are less able to self-renew, less pluripotent, and less able to remain undifferentiated. The degree of their abilities is influenced by the developmental age of the tissue from which they are isolated. For example, stem cells from the placenta, isolated at birth, possess more robust stem-cell-like properties than stem cells isolated from an adult tissue, such as the bone marrow. By the same token, adult stem cells isolated from younger subjects are proving to be more robust than those isolated from older subjects.

\section{WHERE DO WE GET STEM CELLS?}

Embryonic stem cells. As their name suggests, embryonic stem cells are derived from embryos. Specifically, these cells are derived from human eggs that have been fertilized in a laboratory — usually in an in vitro fertilization clinic — and then 
donated for research. They are not derived from eggs fertilized inside a woman's body. Scientists typically derive human embryonic stem cells from embryos that are five days old. Because these cells originate from such an early stage of development, they are believed to be pluripotent. This means that the cells are capable of producing cells of any tissue or organ in the human body. Derivation of human embryonic stem cell lines is controversial because it requires the destruction of the embryo.

Adult stem cells. Adult stem cells are isolated from adult tissues, such as the bone marrow, liver, or brain. Scientists currently believe that many, if not all, adult organs and tissues contain a supply of adult stem cells. As previously described, these adult stem cells serve as a reserve pool of cells for repair and maintenance of the organ in which they reside. A stem cell within the brain, for example, is likely to divide to produce the type of nerve cells and nerve support cells found in the brain. Until recently, it had been thought that an adult stem cell could not produce cells of a very different tissue. However, recent experiments have raised the possibility that stem cells from one tissue may be able to produce cell types of a completely different tissue, a phenomenon known as plasticity or transdifferentiation. Examples of such plasticity include blood cells becoming neurons, liver stem cells that transform their identities to produce insulin, and blood-forming stem cells that can develop into heart muscle cells. Scientists do not agree about whether or not these experiments are valid, and adult stem cell plasticity is a hotly-debated topic. However, the possibility of using adult stem cells for cell-based therapies has become a very active area of scientific investigation. Adult stem cell research does not inspire the controversy that surrounds human embryonic stem cell research because it does not require destruction of an embryo.

Blood forming stem cells (harvested from the bone marrow or the circulating blood) are regularly used to treat human diseases. You can learn more about these types of transplants from the National Marrow Donor Program at this website: http://www.marrow.org/PATIENT/learning_about_bone_ marrow_cord_blood.html.

Stem cells from the placenta and cord blood. Although these two types of stem cells are not technically derived from adult tissues, they are sometimes considered together with adult stem cells. They might more correctly be referred to as "nonembryonic" stem cells, because they are derived from tissue that has developed beyond the embryo stage that concludes at eight weeks postfertilization. Placental and cord blood stem cells are harvested at birth and their collection does not harm the newborn. Since these stem cells originate from tissue that is formed very early in life, scientists believe they may have greater potential for self-renewal and differentiation than adult stem cells, but not as much as human embryonic stem cells. Cord blood stem cells are currently used to treat children with some genetic disorders of the blood and immune system, leukemia and certain cancers, and some inherited metabolic disorders. Until recently, the number of stem cells in a single harvest of cord blood was insufficient to treat adults. However, doctors are now successfully using a new technique that combines more than one sample of cord blood to treat adult patients. Scientists are also attempting to expand cord blood stem cells in laboratory culture, to increase the number of cells available from one harvested batch of cord blood.

\section{SHOULD PARENTS STORE THEIR NEWBORN'S UMBILICAL CORD BLOOD FOR FUTURE USE?}

Each family should make the decision based on their medical history and financial resources. If your family does not have a history of diseases where stem cell transplantation is indicated, it is not likely that your family would use the cord blood cells.

Parents and doctors may find it helpful to consult the report of the American Academy of Pediatrics, from a 1999 work group on cord blood banking: http://aappolicy.aappublications. org/cgi/reprint/pediatrics;104/1/116.pdf. Please be aware that this policy statement was released in 1999, and advances have occurred since its publication.

Currently, cord blood transplants are performed in patients who have blood cancers such as leukemia that cannot be treated with chemotherapy alone or genetic disorders such as sickle cell disease or Cooley's anemia where the patient has not successfully responded to traditional therapies. If doctors decide that such patients need a stem cell transplant, they will determine the most appropriate stem cell source, which may be bone marrow, peripheral blood or cord blood. The cord blood units that are used for transplantation may come from a sibling or an unrelated donor who has a closely matched tissue type. Cord blood transplants rarely occur where the newborn's cord blood is given back to the same donor (autologous transplant). Doctors and patients can learn more about both bone marrow and cord blood transplants from the National Marrow Donor Program's website: http://www.marrow.org/ PATIENT/learning_about_bone_marrow_cord_blood.html.

\section{CHOOSING A CORD BLOOD BANK}

Since cord blood banks are not yet licensed or regulated by the Food and Drug Administration, there isn't a resource available to help consumers make a decision about well established or qualified banks. We suggest that you ask the banks questions such as:

- How many cord blood units stored in your bank have been successfully used in transplantation?

- Have the results of the transplants been reported to a national or international registry?

- Is your bank accredited by professional organizations such as the Foundation for Accreditation of Cellular Therapies or the American Association of Blood Banks?

\section{CAN PARENTS DONATE THEIR NEWBORN'S UMBILICAL CORD BLOOD?}

Although the National Institutes of Health cannot accept donated umbilical cord stem cells from the general public, the National Marrow Donor Program maintains a Web page on 
donating cord blood at http://www.marrow.org/NMDP/ cord_blood_bank_list.html, and the International Cord Blood Society has one at http://www.cordblood.org/public/insights.

\section{WHAT ARE THE ADVANTAGES AND DISADVANTAGES OF USING EMBRYONIC VERSUS NONEMBRYONIC STEM CELLS?}

Human embryonic and nonembryonic stem cells each have advantages and disadvantages with regard to their potential use for treating human beings. As previously mentioned, embryonic stem cells are thought to be pluripotent, while the debate about nonembryonic and adult stem cells' pluripotency is still ongoing, and the state of the scientific possibilities is evolving.

Scientists can grow large numbers of embryonic stem cells in culture with relative ease, while nonembryonic stem cells such as cord blood and placental stem cells may only be collected at birth, and adult stem cells are rare in mature tissues. For many nonembryonic stem cell types, including cord blood stem cells, scientists cannot yet readily expand their numbers in cell culture. This is an important distinction, because large numbers of cells would be needed for stem cell replacement therapies.

At the time techniques for nonembryonic stem cell expansion are developed, however, adult stem cells will present attractive options for scientists. They may be able to expand a patient's own cells and then reintroduce them to treat disease and repair injury. The use of the patient's own cells would mean that the cells would not be rejected by the immune system. This may be a significant advantage over the use of cells or tissues derived from human embryonic stem cells, although researchers have not yet tested whether human recipients would reject tissues derived from donor human embryonic stem cells.

Human embryonic stem cells present a moral dilemma for some, because their derivation requires destruction of a five day old preimplantation stage embryo. Nonembryonic stem cells collected from the placenta and cord blood and those collected from adult tissues do not present this moral dilemma.

\section{WHAT ARE OBSTACLES THAT MUST BE OVERCOME BEFORE STEM CELLS CAN BE USED TO TREAT HUMAN BEINGS?}

There are some types of stem cells that are already being used to treat human beings. Bone marrow research involving human hematopoietic, or blood-forming stem cells, has been pursued in laboratories for more than 50 years. Human cord blood and blood-forming stem cells are used to treat certain conditions. For more information on this, see the previously referenced website of the National Marrow Donor Program: http://www.marrow.org/PATIENT/learning_about_bone_ marrow_cord_blood.html.

We expect that human embryonic stem cell research and other early stem cells will also produce human health benefits, but not until scientists have a firm grasp on the cells' basic biology. Before other types of stem cells can be used to treat patients, scientists must be able to get them to:

Divide and proliferate in the lab to make the required amount of tissue.

Differentiate into the desired cell type(s).

Survive in the recipient after transplant.

Integrate into the surrounding tissue after transplant.

Function appropriately for an extended period of time, optimistically for the duration of the recipient's life.

Remain differentiated, to avoid the risk of cells dividing out of control to form tumors.

Scientists are also experimenting with different research strategies to generate tissues that will not be rejected.

\section{THE FUTURE OF STEM CELL RESEARCH}

There are many possible ways that stem cells may help treat disease and repair tissue damage. The cells themselves may be useful for therapies if scientists become adept at differentiating them into specialized tissues to repair damaged spinal cords, hearts, livers, skin, and other organs. We may also be able to discover what factor(s) give a stem cell its vital properties and use this knowledge to "turn back the clock" on adult cells, recapitulating the pluripotent and self-renewing properties of their progenitor stem cells. This would enable the adult body to repair damage using its own stem cells. Scientists are reporting progress on these and other areas on a daily basis, but there is still a lot of basic research that must be conducted before stem cell therapy becomes commonplace. It is impossible to predict which stem cells or research areas will produce the biggest benefit for a specific clinical approach. Scientists may discover that different types of stem cells or different therapeutic approaches are more suited to treating different diseases. Thus, the National Institutes of Health (NIH) actively supports a wide range of research projects studying many different types of stem cells.

\section{WHERE CAN I GET MORE INFORMATION?}

The NIH stem cell website is a good starting point for basic information about stem cells: http://stemcells.nih.gov/. The NIH site also provides links to other websites with different types of stem cell information. 